\title{
A new clustering approach for group detection in scene-independent dense crowds
}

\begin{abstract}
Despite significant progress in crowd behaviour analysis over the past few years, most of today's state of the art algorithms focus on analysing individual behaviour in a specific-scene. Recently, the widespread availability of cameras and a growing need for public safety have shifted the attention of researchers in video surveillance from individual behavior analysis to group and crowd behavior analysis. However, dangerous and illegal behaviours are mostly occurred from groups of people. Group detection is the main process to separate people in crowded scene into different group based on their interactions. Results of group detection can further to apply in analyze group and crowd behaviour. This paper present a study of the group detection and propose a novel approach for clustering group of people in different crowded scenes based on trajectories. For the clustering of group of people we propose novel formula to compute the weights based on the distance, the occurrence, and the speed correlations of two people in a tracklet cluster to infer the people relationship in a tracklet clusters with Expectation Maximization (EM) in order to overcome occlusion in crowded scenes.
\end{abstract}

Keyword: Group; Group detection; Clustering; Crowded scene; Scene-independent 